\title{
Induction of Immunological Tolerance by Oral Anti-CD3
}

\author{
Andre Pires da Cunha and Howard L. Weiner \\ Center for Neurologic Diseases, Brigham and Women's Hospital, Harvard Medical School, Boston, MA 02115, USA \\ Correspondence should be addressed to Howard L. Weiner, hweiner@rics.bwh.harvard.edu
}

Received 15 May 2011; Accepted 4 September 2011

Academic Editor: Ana Maria Caetano Faria

Copyright () 2012 A. P. da Cunha and H. L. Weiner. This is an open access article distributed under the Creative Commons Attribution License, which permits unrestricted use, distribution, and reproduction in any medium, provided the original work is properly cited.

\begin{abstract}
In recent years, our knowledge about immunoregulation and autoimmunity has significantly advanced, but nontoxic and more effective treatments for different inflammatory and autoimmune diseases are still lacking. Oral tolerance is of unique immunologic importance because it is a continuous natural immunologic event driven by exogenous antigen and is an attractive approach for treatment of these conditions. Parenteral administration of anti-CD3 monoclonal antibody is an approved therapy for transplantation in humans and is effective in autoimmune diabetes. Orally administered anti-CD3 monoclonal antibody is biologically active in the gut and suppresses experimental models of autoimmune diseases. Orally delivered antibody does not have side effects including cytokine release syndromes, thus oral anti-CD3 antibody is clinically applicable for chronic therapy. Here we review findings that identify a novel and powerful immunologic approach that is widely applicable for the treatment of human autoimmune conditions.
\end{abstract}

\section{Introduction}

Understanding how the immune system balances between tolerance and protective immunity is still a key challenge in immunology. Although several approaches have been used to treat autoimmune diseases, they usually involve nonspecific immunosuppression, which frequently comes along with several undesirable side effects. Thus, one of the major goals for the immunotherapy of these pathologies is the induction of regulatory $\mathrm{T}$ cells that mediate immunologic tolerance. In this scenario, the gut environment is particularly important, as tolerance induction is the default immune pathway at this site in physiological conditions [1]. The immunological tolerance to antigens that gain access to the body via the oral route has been termed "oral tolerance" [2] and it has been classically defined as the specific suppression of cellular and/or humoral immune responses to an antigen that was first administered by the oral route $[1,3,4]$.

Studies of oral tolerance have classically involved the administration of oral antigen followed by challenge with the homologous antigen (usually in an adjuvant) to demonstrate antigen-specific tolerance. In this context, an experimental system that has been frequently used for the study of Tcell function in oral tolerance is the use of TCR transgenic
(Tg) mice in which all $\mathrm{T}$ cells have a common TCR. Using TCR Tg mice, we administered the cognate antigens myelin basic protein (MBP) and ovalbumin (OVA) and investigated how oral administration of an antigen-affected specific Tcell subsets. In these studies, we demonstrated the dosedependent induction of Tregs in MBP TCR Tg mice [5] and deletion following high-dose oral administration of OVA in OVA TCR Tg mice [6].

During the course of our experiments, we found that feeding OVA to OVA TCR Tg mice induced CD4+CD25+ Treg cells $[7,8]$. Other investigators also showed that oral antigen induced CD4+CD25+ Tregs [9]. The CD4+ cells from OVA TCR Tg fed animals had greater suppressive properties in vitro than natural Tregs, mediated suppression in part by both TGF- $\beta$ and IL-10, and presented increased expression of CTLA-4, a molecule known to be involved in Treg activity $[6,10]$. Although, these findings demonstrated that oral antigen could induce/expand Tregs, administration of OVA to OVA TCR Tg mice is dependent on TCR Tg mice and not translatable to humans. We thus asked whether it was possible to trigger the TCR in the gut CD4+ T cells of wild-type mice and induce Tregs without using cognate antigen. This possibility will be discussed in more detail below. 


\section{Immune Tolerance and Anti-CD3 Antibody Treatment}

Different mechanisms have been implicated in the induction and maintenance of immune tolerance including deletion, anergy, and active cellular regulation [11]. One approach that has been successfully used for the induction/restoration of immune tolerance is the administration of CD3-specific antibody. Different groups have demonstrated that parenteral administration of anti-CD3 is effective not only in animal models of autoimmunity, including autoimmune diabetes [12-14] and experimental autoimmune encephalomyelitis (EAE) $[15,16]$ but also in human trials of autoimmune diabetes [17-19] and psoriatic arthritis [20]. Furthermore, intravenous administration of anti-CD3 is an approved therapy for acute transplant rejection in humans although side effects related to cytokines release limit its chronic use $[21,22]$. Humanized antibodies have been designed to reduce these side effects $[23,24]$ but the successful translation might require new therapies that would be more physiologic and less toxic and mucosal tolerance can be exploited in this direction.

2.1. Oral Anti-CD3. It is known that anti-CD3 binds to the $\varepsilon$ chain of the TCR complex and when given intravenously deletes $\mathrm{T}$ cells and, as mentioned above, has been shown to be an effective treatment for type 1 diabetes in the nonobese diabetic (NOD) mouse [25]. We hypothesized that oral administration of anti-CD3 monoclonal antibody would replace the use of a cognate antigen to trigger the TCR and would thus induce Tregs when given orally. Monoclonal antibodies have not been given orally on the assumption that they would be degraded in the gut and thus would not be biologically active. Nonetheless, it is known that orally administered cytokines [26] and peptides $[27,28]$ are biologically active, demonstrating that orally administered proteins are not completely degraded in the gut.

Thus, to test this hypothesis, we administered hamster anti-mouse CD3 (2C11 clone) to SJL mice and immunized with PLP/CFA to induce EAE. We found that oral anti$\mathrm{CD} 3$ suppressed both clinical and pathologic features of EAE both in the PLP and MOG EAE model [29]. There was a dose effect observed, with EAE suppression by oral anti-CD3 seen at lower $(5 \mu \mathrm{g})$, but not higher doses $(50 \mu \mathrm{g}, 500 \mu \mathrm{g})$. These findings were consistent with the classic paradigm of oral tolerance in which induction of Tregs is seen at lower but not higher doses [1]. Furthermore, it demonstrates that induction of Tregs by oral anti-CD3 is not simply related to administering large amounts of antibody to overcome degradation of the antibody in the gut. Indeed, biologically active anti-CD3 could be isolated from intestinal eluates of animals that were given anti-CD3 orally [30] and we could also visualize anti-CD3 being taken up by gut epithelial cells and binding to gut DCs in intestinal loop experiments [1]. The extent to which these DCs that take up anti-CD3 play a role in the mechanism of action of oral anti-CD3 has still to be better studied. Of note, the Fc portion of anti-CD3 was not required, as anti-CD3 Fab' 2 fragment is active orally and induces Tregs.
2.2. Differences between Intravenous and Oral Anti-CD3 Antibody. It is known that intravenous anti-CD3 enters the blood stream, modulates $\mathrm{CD} 3$ from the cell surface, and leads to the depletion of $\mathrm{CD} 3+\mathrm{T}$ cells. Oral anti-CD3, on the other hand, does not enter the blood stream or modulate CD3 from the cell surface but acts locally in the gut to induce Th3 type CD4+CD25-LAP+ Tregs in the MLNs. As oral anti-CD3 does not enter the bloodstream, there is no cytokine release syndrome, one of the main problems with the intravenous administration of anti-CD3. In the EAE model, intravenous anti-CD3 is effective when given after disease manifests but not when given prior to disease induction. Oral anti-CD3, on the other hand, ameliorates EAE both when given prior to EAE induction and at the height of disease [29]. The explanation for this difference is related to the fact that intravenous anti-CD3 acts primarily by lysing disease effector cells (present only after disease induction), whereas oral anti-CD3 acts by inducing Tregs. Intravenous anti-CD3 has been reported to induce Tregs that act in a TGF- $\beta$-dependent fashion, but only after lysis of $\mathrm{T}$ cells [12]. Therefore, immunoregulation by oral antiCD3 involves different mechanisms other than intravenous anti-CD3 and has the advantage of being safer as it is not associated with systemic side effects [31].

We found that oral anti-CD3 ameliorates disease in other autoimmune and inflammatory diseases including streptozocin-induced [30] and NOD autoimmune diabetes (unpublished data), type 2 diabetes in the $\mathrm{Ob} / \mathrm{Ob}$ mouse [32], lupus prone SNF1mice [33], and collagen-induced arthritis [34]. A different group of investigators reported that oral anti-CD3 suppresses atherosclerosis in $\mathrm{ApoE}^{-/-}$ mice [35]. In all these models, disease amelioration was related to the induction of TGF- $\beta$-dependent Tregs that express LAP on their surface. Indeed, in vivo treatment of animals with anti-LAP antibody [36], abrogated tolerance induction by oral anti-CD3 in the EAE model (da Cunha et al. [1], unpublished). We also found that nasal anti-CD3 ameliorates lupus but does so by inducing an IL10-dependent CD4+CD25-LAP+ Treg as opposed to the TGF- $\beta$-dependent LAP + Treg induced by oral anti-CD3 [37]. This is consistent with the observation that GALT DCs induce TGF- $\beta$-dependent Tregs versus IL-10-dependent Tregs induced in the bronchial associated lymphoid tissue [38]. Furthermore, these results demonstrate that mucosally administered anti-CD3 appears to act in a fashion analogous to mucosally administered cognate antigen [38].

The effects of oral anti-CD3 raise the question whether it is more advantageous to induce antigen-specific versus antigen nonspecific Tregs for the treatment of autoimmune and inflammatory diseases. It is assumed that the induction of antigen-specific Tregs is preferable, as one would have specific immune modulation with less potential side effects. Furthermore, because of the phenomenon of bystander suppression, which we first described in association with oral tolerance [39], cytokines such as TGF- $\beta$ released from antigen-specific Tregs at the target organ would suppress reactivity to other autoantigens that developed in the course of epitope spreading. Of note, there may be target organ specificity even when antigen nonspecific Tregs are induced 
with oral anti-CD3 as we observed increased numbers of Th3 type LAP+ Tregs in the pancreatic lymph nodes of autoimmune diabetic mice [30], and this has been suggested for atherosclerosis models [29]. Furthermore, in conditions such as type 2 diabetes, lupus, and atherosclerosis, there are not well-defined target antigens and in these conditions induction of antigen nonspecific Tregs by anti-CD3 may be preferable. Studies are in progress to test the combination of mucosal anti-CD3 given with antigen. As discussed below, oral anti-CD3 is currently being tested in humans.

\section{Oral Anti-CD3 in Humans}

Oral anti-CD3 has been tested in a phase 1 study in healthy human volunteers (3 per group) who were orally administered $0.2,1.0$, or $5.0 \mathrm{mg}$ of mouse anti-human OKT3 mAb daily for 5 days [31]. Immunologic effects were observed in the peripheral blood and consisted of transient proliferation, suppression of Th1/Th17 responses, increased expression of Treg markers, increased TGF- $\beta /$ IL-10, and decreased IL-23/IL-6 expression in dendritic cells. No side effects were observed. There were no human anti-mouse antibody responses, changes in CD3 cells in the blood, or modulation of CD3 from the surface of $\mathrm{T}$ cells. The optimal dose was found to be $1 \mathrm{mg}$.

Oral OKT3 mAb has also been recently tested in a single-blind randomized placebo-controlled phase 2 a study in patients with nonalcoholic steatohepatitis (NASH) and altered glucose metabolism that included subjects with type2 diabetes [40]. The study was performed at the HadassahHebrew University Medical Center in Jerusalem, Israel. OKT3 or placebo was orally administered (9 per group) at doses of $0.2,1.0$, and $5.0 \mathrm{mg}$. In the NASH study 36 subjects were treated once daily for 30 days with final follow-up 60 days after the first dose.

Oral OKT3 was safe with no adverse effects or systemic toxicity as measured by blood hematology, chemistry, immunological safety markers, and physical signs. There were no changes in blood levels of CD3, CD4, or CD8positive cells. Oral OKT3 induced regulatory $\mathrm{T}$ cells, which generally persisted to day 60 and trends in cytokine production consistent with effects observed in the phase 1 clinical study and in animal models. Positive trends in clinical parameters, some of which were statistically significant were also observed including a reduction in liver enzymes and reduced blood levels of glucose and insulin. Several of the positive efficacy trends persisted to day 60 following cessation of treatment at day 30 . Some subjects had increased levels of serum antibodies directed against OKT3, which did not affect the positive trial results observed. These results suggest that oral anti-CD3 mAb may have clinical benefit for subjects with NASH or type- 2 diabetes. Confirmatory studies are now needed, including studies with humanized antibodies. These results will provide the basis for investigating oral/nasal antiCD3 in other autoimmune and inflammatory conditions in humans.

Recently, an important development that will facilitate studies related to the use of humanized anti-CD3 antibodies was the generation of transgenic mice expressing the $\varepsilon$ chain of the human CD3 complex on the NOD background $(\mathrm{NOD}-\mathrm{huCD} 3 \varepsilon)$. These mice develop spontaneous diabetes to the same extent as the wild-type NOD mice and when treated with a humanized anti-CD3 antibody, they exhibited a complete and durable disease remission [41]. This model will be very useful for studies evaluating anti-human CD3 antibodies, including its administration by oral route, and this will open new perspectives and clarify their potential clinical utility.

\section{Summary}

Mucosal tolerance is an attractive approach for the treatment of autoimmune inflammatory diseases as it is a clinically applicable physiologic manner to suppress inflammation through the induction of regulatory $\mathrm{T}$ cells. Its lack of toxicity and ease of administration are also important features desired for an effective immunotherapy. Several studies have demonstrated that oral (or nasal) administration of anti-CD3 monoclonal antibodies can be used to induce immune tolerance and is effective in the treatment of animal models of autoimmune diseases. These studies have also demonstrated that mucosal administration of anti-CD3 antibodies does not lead to detectable side effects including cytokine release syndrome or antiglobulin response and thus could be used as a continuous therapy for the treatment of these conditions. In summary, results presented here indicate that oral administration of anti-CD3 antibodies represents a new avenue that can be investigated for the treatment of autoimmune diseases.

\section{References}

[1] H. L. Weiner, A. P. da Cunha, F. Quintana, and H. Wu, "Oral tolerance," Immunological Reviews, vol. 241, no. 1, pp. 241259, 2011.

[2] N. M. Vaz, L. C. S. Maia, D. G. Hanson, and J. M. Lynch, "Inhibition of homocytotropic antibody responses in adult inbred mice by previous feeding of the specific antigen," Journal of Allergy and Clinical Immunology, vol. 60, no. 2, pp. 110-115, 1977.

[3] A. M. C. Faria and H. L. Weiner, "Oral tolerance: therapeutic implications for autoimmune diseases," Clinical and Developmental Immunology, vol. 13, no. 2-4, pp. 143-157, 2006.

[4] S. Strobel and A. M. Mowat, "Oral tolerance and allergic responses to food proteins," Current Opinion in Allergy and Clinical Immunology, vol. 6, no. 3, pp. 207-213, 2006.

[5] Y. Chen, J. I. Inobe, V. K. Kuchroo, J. L. Baron, C. A. Janeway, and H. L. Weiner, "Oral tolerance in myelin basic protein T-cell receptor transgenic mice: suppression of autoimmune encephalomyelitis and dose-dependent induction of regulatory cells," Proceedings of the National Academy of Sciences of the United States of America, vol. 93, no. 1, pp. 388-391, 1996.

[6] Y. Chen, J. I. Inobe, R. Marks, P. Gonnella, V. K. Kuchroo, and H. L. Weiner, "Peripheral deletion of antigen-reactive T cells in oral tolerance," Nature, vol. 376, no. 6536, pp. 177-180, 1995.

[7] C. M. Sun, J. A. Hall, R. B. Blank et al., "Small intestine lamina propria dendritic cells promote de novo generation of Foxp3 T reg cells via retinoic acid," Journal of Experimental Medicine, vol. 204, no. 8, pp. 1775-1785, 2007. 
[8] X. Zhang, L. Izikson, L. Liu, and H. L. Weiner, "Activation of $\mathrm{CD} 25^{+} \mathrm{CD} 4^{+}$regulatory $\mathrm{T}$ cells by oral antigen administration," Journal of Immunology, vol. 167, no. 8, pp. 4245-4253, 2001.

[9] K. M. Thorstenson and A. Khoruts, "Generation of anergic and potentially immunoregulatory $\mathrm{CD} 25^{+} \mathrm{CD} 4 \mathrm{~T}$ cells in vivo after induction of peripheral tolerance with intravenous or oral antigen," Journal of Immunology, vol. 167, no. 1, pp. 188195, 2001

[10] K. Wing, Y. Onishi, P. Prieto-Martin et al., "CTLA-4 control over Foxp3+ regulatory T cell function," Science, vol. 322, no. 5899, pp. 271-275, 2008.

[11] J. A. Bluestone, "Mechanisms of tolerance," Immunological Reviews, vol. 241, no. 1, pp. 5-19, 2011.

[12] M. Belghith, J. A. Bluestone, S. Barriot, J. Mégret, J. F. Bach, and L. Chatenoud, "TGF- $\beta$-dependent mechanisms mediate restoration of self-tolerance induced by antibodies to CD3 in overt autoimmune diabetes," Nature Medicine, vol. 9, no. 9, pp. 1202-1208, 2003.

[13] L. Chatenoud, E. Thervet, J. Primo, and J. F. Bach, "Anti-CD3 antibody induces long-term remission of overt autoimmunity in nonobese diabetic mice," Proceedings of the National Academy of Sciences of the United States of America, vol. 91, no. 1, pp. 123-127, 1994.

[14] K. C. Herold, J. A. Bluestone, A. G. Montag et al., "Prevention of autoimmune diabetes with nonactivating anti-CD3 monoclonal antibody," Diabetes, vol. 41, no. 3, pp. 385-391, 1992.

[15] A. P. Kohm, J. S. Williams, A. L. Bickford et al., "Treatment with nonmitogenic anti-CD3 monoclonal antibody induces CD4+ T cell unresponsiveness and functional reversal of established experimental autoimmune encephalomyelitis," Journal of Immunology, vol. 174, no. 8, pp. 4525-4534, 2005.

[16] G. T. Tran, N. Carter, X. Y. He et al., "Reversal of experimental allergic encephalomyelitis with non-mitogenic, non-depleting anti-CD3 mAb therapy with a preferntial effect on Th1 cells that is augmented by IL-4," International Immunology, vol. 13, no. 9, pp. 1109-1120, 2001.

[17] K. C. Herold, S. E. Gitelman, U. Masharani et al., "A single course of anti-CD3 monoclonal antibody hOKT3 $\gamma 1$ (ala-ala) results in improvement in C-peptide responses and clinical parameters for at least 2 years after onset of type 1 diabetes," Diabetes, vol. 54, no. 6, pp. 1763-1769, 2005.

[18] K. C. Herold, W. Hagopian, J. A. Auger et al., "Anti-CD3 monoclonal antibody in new-onset type 1 diabetes mellitus," New England Journal of Medicine, vol. 346, no. 22, pp. 1692 1698, 2002.

[19] K. C. Herold and L. Taylor, "Treatment of type 1 diabetes with anti-CD3 monoclonal antibody: induction of immune regulation?" Immunologic Research, vol. 28, no. 2, pp. 141-150, 2003.

[20] T. O. Utset, J. A. Auger, D. Peace et al., "Modified anti-CD3 therapy in psoriatic arthritis: a Phase I/II clinical trial," Journal of Rheumatology, vol. 29, no. 9, pp. 1907-1913, 2002.

[21] L. Chatenoud, "CD3-specific antibody-induced active tolerance: from bench to bedside," Nature Reviews Immunology, vol. 3, no. 2, pp. 123-132, 2003.

[22] L. Chatenoud, "CD3-specific antibodies as promising tools to aim at immune tolerance in the clinic," International Reviews of Immunology, vol. 25, no. 3-4, pp. 215-233, 2006.

[23] M. L. Alegre, L. J. Peterson, D. Xu et al., "A non-activating 'humanized' anti-CD3 monoclonal antibody retains immunosuppressive properties in vivo," Transplantation, vol. 57, no. 11, pp. 1537-1543, 1994.
[24] S. Bolt, E. Routledge, I. Lloyd et al., "The generation of a humanized, non-mitogenic CD3 monoclonal antibody which retains in vitro immunosuppressive properties," European Journal of Immunology, vol. 23, no. 2, pp. 403-411, 1993.

[25] L. Chatenoud and J. A. Bluestone, "CD3-specific antibodies: a portal to the treatment of autoimmunity," Nature Reviews Immunology, vol. 7, no. 8, pp. 622-632, 2007.

[26] A. J. Slavin, R. Maron, and H. L. Weiner, "Mucosal administration of IL-10 enhances oral tolerance in autoimmune encephalomyelitis and diabetes," International Immunology, vol. 13, no. 6, pp. 825-833, 2001.

[27] K. Miyamoto, C. I. Kingsley, X. Zhang et al., "The ICOS molecule plays a crucial role in the development of mucosal tolerance," Journal of Immunology, vol. 175, no. 11, pp. 73417347, 2005.

[28] J. P. S. Peron, K. Yang, M. L. Chen et al., "Oral tolerance reduces Th17 cells as well as the overall inflammation in the central nervous system of EAE mice," Journal of Neuroimmunology, vol. 227, no. 1-2, pp. 10-17, 2010.

[29] H. Ochi, M. Abraham, H. Ishikawa et al., "Oral CD3specific antibody suppresses autoimmune encephalomyelitis by inducing $\mathrm{CD} 4^{+} \mathrm{CD} 25^{-} \mathrm{LAP}^{+} \mathrm{T}$ cells," Nature Medicine, vol. 12, no. 6, pp. 627-635, 2006.

[30] H. Ishikawa, H. Ochi, M. L. Chen, D. Frenkel, R. Maron, and H. L. Weiner, "Inhibition of autoimmune diabetes by oral administration of anti-CD3 monoclonal antibody," Diabetes, vol. 56, no. 8, pp. 2103-2109, 2007.

[31] Y. Ilan, E. Zigmond, G. Lalazar et al., "Oral administration of OKT3 monoclonal antibody to human subjects induces a dose-dependent immunologic effect in T cells and dendritic cells," Journal of Clinical Immunology, vol. 30, no. 1, pp. 167177, 2010.

[32] Y. Ilan, R. Maron, A. M. Tukpah et al., "Induction of regulatory $\mathrm{T}$ cells decreases adipose inflammation and alleviates insulin resistance in ob/ob mice," Proceedings of the National Academy of Sciences of the United States of America, vol. 107, no. 21, pp. 9765-9770, 2010.

[33] H. Y. Wu, E. M. Center, G. C. Tsokos, and H. L. Weiner, "Suppression of murine SLE by oral anti-CD3: inducible $\mathrm{CD}^{+} \mathrm{CD} 25^{-} \mathrm{LAP}^{+}$regulatory $\mathrm{T}$ cells control the expansion of IL-17+ follicular helper T cells," Lupus, vol. 18, no. 7, pp. 586596, 2009.

[34] H. Y. Wu, R. Maron, A. M. Tukpah, and H. L. Weiner, "Mucosal anti-CD3 monoclonal antibody attenuates collageninduced arthritis that is associated with induction of LAP+ regulatory $\mathrm{T}$ cells and is enhanced by administration of an emulsome-based Th2-skewing adjuvant," Journal of Immunology, vol. 185, no. 6, pp. 3401-3407, 2010.

[35] N. Sasaki, T. Yamashita, M. Takeda et al., "Oral anti-CD3 antibody treatment induces regulatory $\mathrm{t}$ cells and inhibits the development of atherosclerosis in mice," Circulation, vol. 120, no. 20, pp. 1996-2005, 2009.

[36] T. Oida and H. L. Weiner, "Overexpression of TGF- $\beta 1$ gene induces cell surface localized glucose-regulated protein 78 -associated latency-associated peptide/TGF- $\beta$," Journal of Immunology, vol. 185, no. 6, pp. 3529-3535, 2010.

[37] H. Y. Wu, F. J. Quintana, and H. L. Weiner, "Nasal antiCD3 antibody ameliorates lupus by inducing an IL-10secreting $\mathrm{CD}^{+} \mathrm{CD}_{25}{ }^{-} \mathrm{LAP}^{+}$regulatory $\mathrm{T}$ Cell and is associated with down-regulation of $\mathrm{IL}_{-}-17^{+} \mathrm{CD} 4^{+} \mathrm{ICOS}^{+} \mathrm{CXCR} 5^{+}$ follicular helper T cells," Journal of Immunology, vol. 181, no. 9, pp. 6038-6050, 2008. 
[38] H. L. Weiner, "The mucosal milieu creates tolerogenic dendritic cells and TR 1 and TH3 regulatory cells," Nature Immunology, vol. 2, no. 8, pp. 671-672, 2001.

[39] A. Miller, O. Lider, and H. L. Weiner, "Antigen-driven bystander suppression after oral administration of antigens," Journal of Experimental Medicine, vol. 174, no. 4, pp. 791-798, 1991.

[40] NasVax, "NasVax Announces the Success of a Phase 2a Clinical Trial of a New Oral Immunotherapy for Fatty Liver Disease," 2011, http://www.businesswire.com/news/home/ 20110321005934/en/NasVax-Announces-Success-Phase-2aClinical-Trial.

[41] C. Kuhn, S. You, F. Valette et al., "Human CD3 transgenic mice: preclinical testing of antibodies promoting immune tolerance," Science Translational Medicine, vol. 3, no. 68, article 68ra10, 2011. 


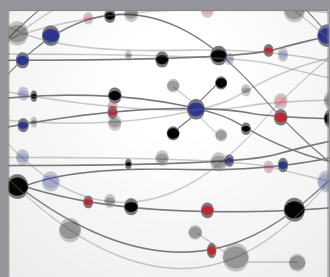

The Scientific World Journal
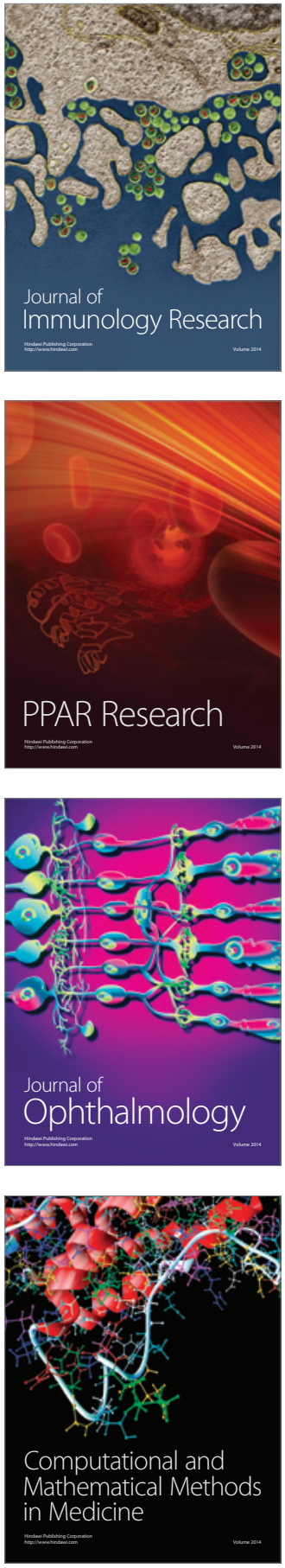

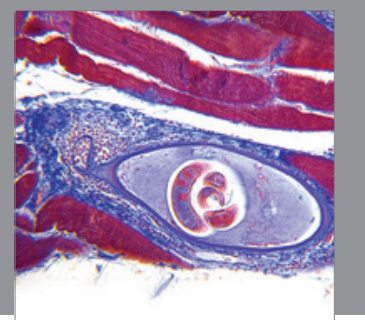

Gastroenterology

Research and Practice
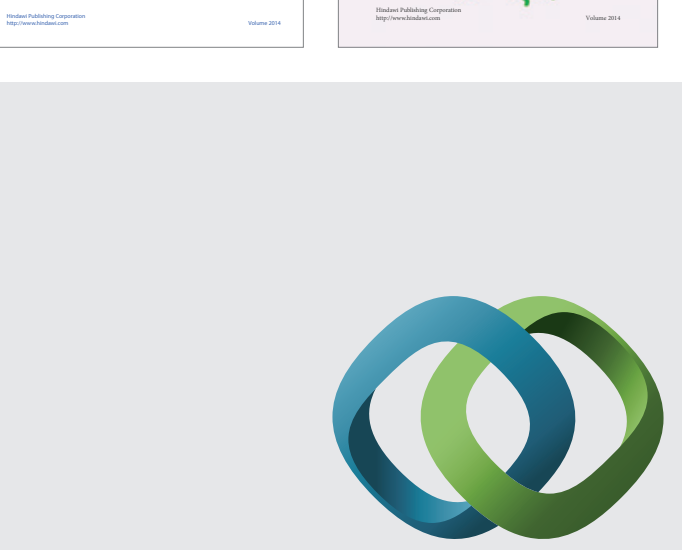

\section{Hindawi}

Submit your manuscripts at

http://www.hindawi.com
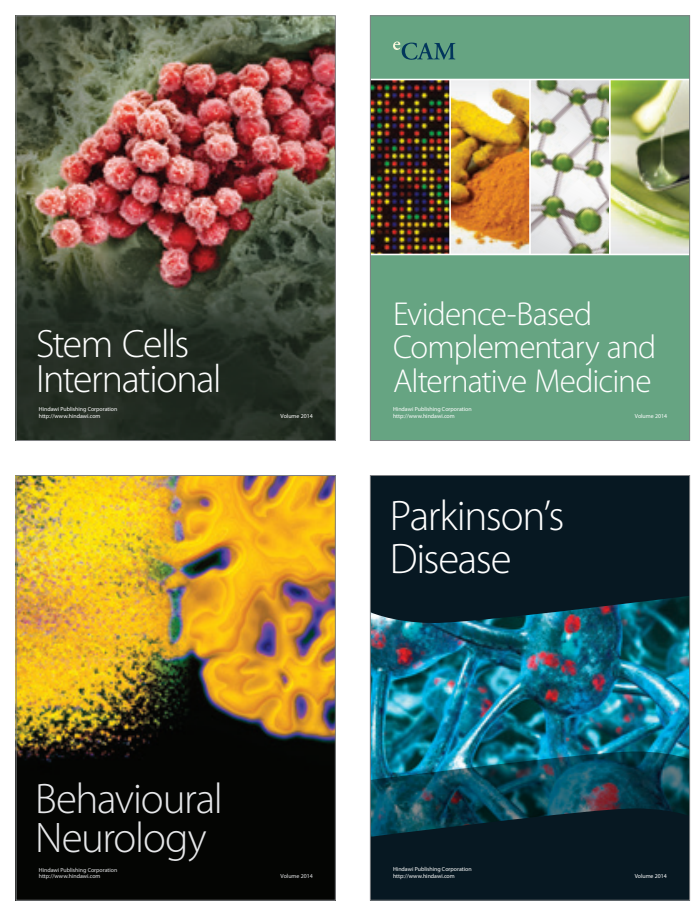

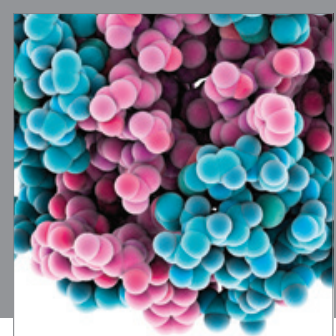

Journal of
Diabetes Research

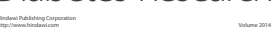

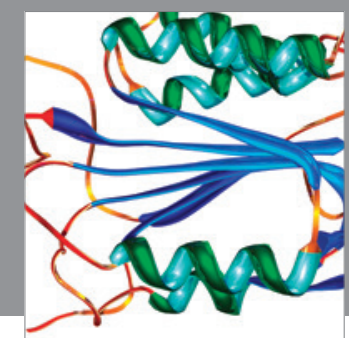

Disease Markers
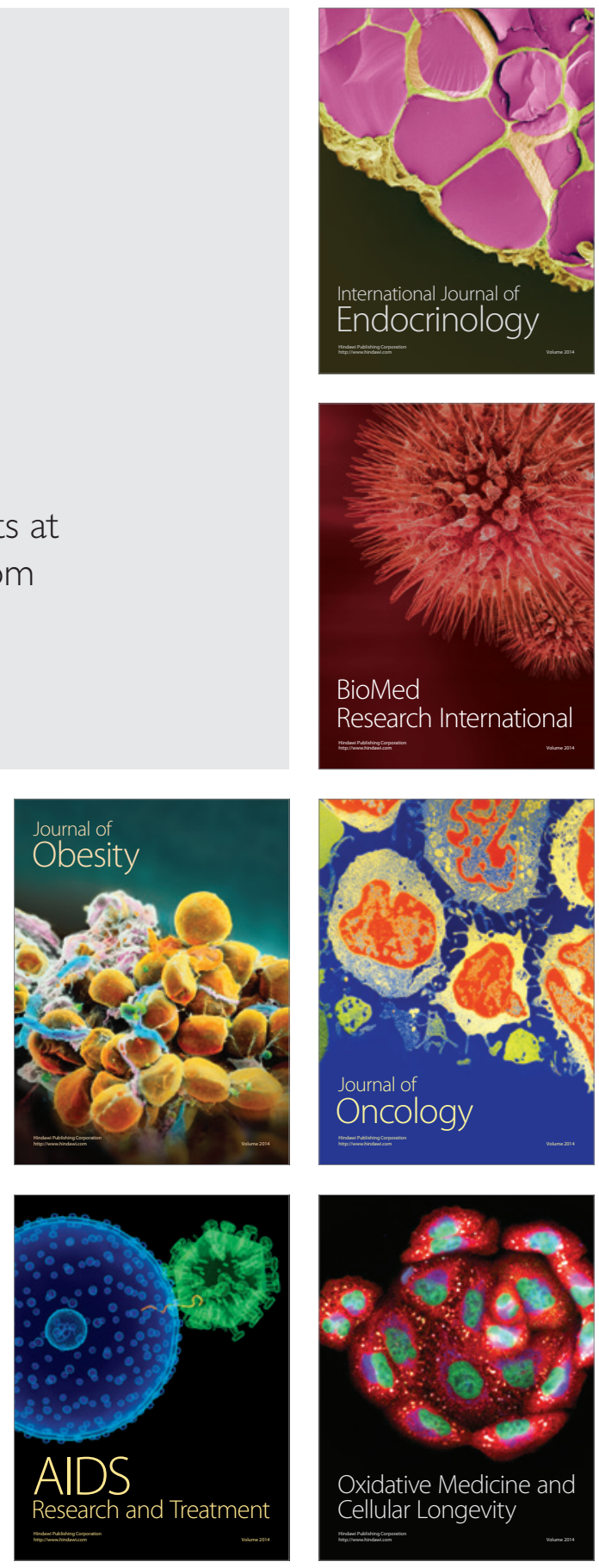